\title{
Análise das experiências da Comunidade de Práticas sobre o enfrentamento do câncer de colo de útero
}

César Augusto Paro ${ }^{1}$, Lívia Moura de Sousa ${ }^{2}$, Neide Emy Kurokawa e Silva ${ }^{3}$

\section{Resumo}

Este trabalho buscou analisar as ações desenvolvidas para a promoção da saúde e prevenção no enfrentamento do câncer de colo de útero. Tratou-se de uma pesquisa documental e exploratória do tipo qualitativa. Foram coletados relatos de experiências publicados por profissionais da saúde na Comunidade de Práticas. A análise pautouse na identificação das principais tendências pedagógicas na educação em saúde: de transmissão de informações, de condicionamento e de problematização crítica. A amostra contou com 15 relatos desenvolvidos predominantemente pelo público feminino, com a tônica no exame preventivo, em unidades de atenção primária à saúde e com ações pontuais no modelo de campanha. A maioria das ações partiu de uma concepção pedagógica de transmissão, visando o repasse de informações acerca da doença e de seu tratamento e prevenção, seguido de ações que se utilizaram do condicionamento, com atividades que visavam alguma recompensa para o objetivo pretendido. A menor parte delas atuaram numa lógica da problematização, visando atuar a partir da perspectiva dialógicareflexiva. A tímida inserção de atividades com uma abordagem crítica sugere o quanto o ideário da promoção da saúde e da educação popular em saúde merece ser fortalecido nos serviços de saúde.

\section{Palavras-chave}

Câncer de colo de útero. Promoção da Saúde. Prevenção de Doenças. Atenção Primária à Saúde. Educação em Saúde.

${ }^{1}$ Doutorando em Saúde Coletiva na Universidade Federal do Rio de Janeiro, Brasil, com período sanduíche na Seção de Saúde Global da Universidade de Copenhagen, Dinamarca; integrante do Grupo Temático de Educação Popular e Saúde da Associação Brasileira de Saúde Coletiva. E-mail: cesaraugustoparo@iesc.ufrj.br.

2 Especialista em Processos Educativos Gestão Regionalizada do SUS pela Escola de Saúde Pública da Bahia, Brasil. E-mail: liviamouras@gmail.com.

3 Doutora em Medicina pela Universidade de São Paulo, Brasil; professora adjunta no Instituto de Estudos em Saúde Coletiva da Universidade Federal do Rio de Janeiro, Brasil. E-mail: neks@iesc.ufrj.br. 


\title{
An analysis of the Community's experiences in practices for the uterine cervical neoplasm control
}

César Augusto Paro ${ }^{4}$, Lívia Moura de Sousa ${ }^{5}$, Neide Emy Kurokawa e Silva ${ }^{6}$

\begin{abstract}
This paper aimed to analyze actions developed to health promotion and disease prevention in the uterine cervical neoplasm control. It was a documentary, exploratory and qualitative research. Experience reports published in Practice Community were collected to be analyzed. They were analyzed by content analysis with three pedagogical perspectives: pedagogy of transmission, behaviorist pedagogy, and problematizing critical pedagogy. 15 experience reports were selected. These experiences were developed predominantly by the female public, about the preventive examination Pap, inside health care centers, and punctual actions by campaign model. There were prevailed actions by the pedagogy of transmission that aimed to transmit knowledge about diseases and its treatment and prevention. Secondary, there were actions in a behaviorist perspective that aimed to give some reward to a determinate response. The minority of the actions adopted a problematization perspective that developed dialogical and reflexives activities. These results showed the necessity to strengthen the health promotion and the popular education in health in the healthcare services.
\end{abstract}

\section{Keywords}

Uterine Cervical Neoplasms. Health Promotion. Disease Prevention. Primary Health Care. Health Education.

\footnotetext{
${ }^{4} \mathrm{PhD}$ student in Collective Health, Federal University of Rio de Janeiro, Brazil, with a sandwich period at the Global Health Section of the University of Copenhagen, Denmark; member of the Thematic Group of Popular Health Education, Brazilian Association of Collective Health. E-mail: cesaraugustoparo@iesc.ufrj.br.

5 Specialist in Educational Processes Regionalized Management of SUS, School of Public Health of Bahia, Brazil. E-mail: liviamouras@gmail.com.

${ }^{6} \mathrm{PhD}$ in Medicine, University of São Paulo, Brazil; adjunct professor at the Institute of Public Health Studies at the Federal University of Rio de Janeiro, Brazil. E-mail: neks@iesc.ufrj.br.
} 


\section{Introdução}

As ações de promoção da saúde e de prevenção de doenças são atividades previstas principalmente para serem desenvolvidas no primeiro nível de atenção da rede de saúde (KUSCHNIR, 2015), compreendidas na própria conceituação de cuidados primários de saúde (BUSS, 2000). Essas ações são importantes no enfrentamento do câncer de colo de útero, uma vez que este é considerado um dos tipos de câncer que tem maior potencial de prevenção, com $100 \%$ de possibilidade de cura dos casos diagnosticados em sua fase inicial (KUSCHNIR; SILVA, 2015).

Nesse sentido, o Programa Nacional de Controle do Câncer do Colo do Útero aponta a necessidade de ações de promoção da saúde que atuem sobre os determinantes sociais do processo saúde-doença e promovam qualidade de vida, a prevenção primária com vistas a diminuição do risco de contágio do Papiloma Vírus Humano (HPV) e a própria realização do exame de detecção precoce na população alvo (INCA, 2011). Alguns fatores de risco também devem ser trabalhados no seu enfrentamento, como o tabagismo, a coinfecção por outras infecções sexualmente transmissíveis (IST), a iniciação sexual precoce, a multiplicidade de parceiros sexuais, a multiparidade, o uso prolongado de contraceptivos orais e a alimentação não saudável (WHO, 2014).

Para que o controle do câncer de colo de útero seja efetivo, as ações de promoção deveriam fortalecer o eixo de mobilização social para o conhecimento e o debate público dos fatores relacionados à ocorrência desse câncer. Já as ações de prevenção deveriam se dar em um contexto educativo que privilegiasse a interação, a participação e o diálogo, com acesso à informação sobre redução da exposição aos riscos e formas de proteção articulada a um conjunto de fatores relacionados a oportunidades para práticas saudáveis e acesso a serviços de qualidade (PARADA et al., 2008). Nesse sentido, tais ações se coadunariam com uma perspectiva pedagógica pautada em uma educação libertadora, que visasse à transformação social (FREIRE, 2016).

Apesar de diversos dos fatores relacionados a esse câncer serem conhecidos, a efetividade das ações de promoção e prevenção no seu enfrentamento ainda é um desafio para os serviços que compõem a rede de atenção à saúde no Sistema Único de Saúde (SUS), trazendo indagações quanto aos insucessos dessas iniciativas. Considerando que as práticas pedagógicas subjacentes às ações de promoção e de prevenção podem ter importante papel na mitigação das vulnerabilidades da população ao câncer, o objetivo deste artigo é analisar as ações educativas desenvolvidas para o enfrentamento do câncer de colo de útero registradas 
na Comunidade de Práticas (CdP), caracterizando-as e analisando as perspectivas pedagógicas adotadas.

\section{Metodologia}

Tratou-se de uma pesquisa documental e exploratória do tipo qualitativa (LAVILLE; DIONNE, 1999), a partir dos relatos de experiências relacionados às ações de promoção da saúde e prevenção ao enfrentamento do câncer de colo de útero publicados por profissionais da saúde na CdP.

A CdP é uma rede social institucional criada do Ministério da Saúde (MS) brasileiro, figurando como um espaço de encontro e compartilhamento de experiências de gestores e trabalhadores da saúde com a finalidade de formar uma rede colaborativa voltada para a melhoria das condições de trabalho e da qualidade do cuidado à saúde da população. Trata-se de uma plataforma eletrônica (https://novo.atencaobasica.org.br/) que, dentre suas principais funcionalidades, permite trocar experiências no interior de comunidades, realizar cursos e "amostrar-se" para os demais usuários.

Na seção de relatos de experiência, há um banco de dados de relatos cadastrados por profissionais de gestão de saúde que descrevem vivências profissionais tidas como exitosas ou não e que contribuam com a discussão, troca e proposição de ideias para a melhoria do cuidado em saúde. Desde a criação dessa plataforma em 2011, já houve o cadastro de 7.880 relatos (dado atualizado em 01/09/2017).

As experiências foram selecionadas a partir da leitura na íntegra dos relatos que utilizaram os termos "câncer de colo de útero", "colo de útero" ou "útero" como descritores. Nesse estudo, foram incluídos somente aquelas que registrassem ações relativas à promoção da saúde e prevenção no enfrentamento do câncer de colo de útero. Elas foram descritas e caracterizadas a partir de seus objetivos, estratégias metodológicas, organizadores da ação, público-alvo, localidade e duração.

Para analisar as perspectivas pedagógicas presentes na ação desenvolvida, foi utilizada a análise do conteúdo (MINAYO, 2014). O processo de tratamento dos dados nessa etapa iniciou-se com a pré-análise, fase em que se realizou a preparação do material e leituras dos achados, a fim de propiciar o contato exaustivo com os dados e consequente impregnação pelo conteúdo. Posteriormente, realizou-se a exploração do material por meio da operação de codificação, com o recorte dos dados e sua compilação em unidades de significados (categorias e subcategorias), para, enfim, os dados serem descritos e interpretados. 


\section{Resultados e Discussão}

Dos 7.880 relatos presentes na CdP no momento da coleta de dados (setembro de 2017), 71 utilizaram os termos "câncer de colo de útero", "colo de útero" ou "útero" como descritores. Na leitura desses relatos, foram excluídos $56(78,9 \%)$ por não se relacionarem com o escopo da pesquisa: 24 (42,9\%) apresentavam diagnósticos de saúde relacionados ao câncer de colo de útero e/ou da oferta de serviços para o seu enfrentamento, 9 (16,1\%) traziam iniciativas de gestão do trabalho e do cuidado de serviços que realizavam alguma ação no cuidado integral para esse câncer, 7 (12,5\%) relatavam estratégias de educação permanente para os profissionais de saúde envolvidos no enfrentamento dessa doença e 16 $(28,6 \%)$ não tinham relação direta com o cuidado ao câncer de colo de útero. 15 relatos $(21,1 \%)$ apresentavam ações relativas à promoção da saúde e prevenção no enfrentamento do câncer de colo de útero, incluídos, portanto, como corpus desse estudo. O Quadro 1 apresenta uma síntese da caracterização desses relatos.

Quadro 1 - Relatos de experiência de promoção da saúde e prevenção no enfrentamento do câncer de colo de útero

\begin{tabular}{|c|c|c|c|c|c|c|}
\hline Título & Objetivo & Metodologia & Organização & Público-alvo & Localidade & Duração \\
\hline $\begin{array}{l}\text { A conscientização } \\
\text { da mulher para } \\
\text { fazer prevenção } \\
\text { contínua ao câncer } \\
\text { de colo de útero }\end{array}$ & $\begin{array}{l}\text { Proporcionar } \\
\text { conhecimento } \\
\text { nas mulheres } \\
\text { para que } \\
\text { desenvolvam } \\
\text { compromisso } \\
\text { individual em } \\
\text { realizar o } \\
\text { exame } \\
\text { preventivo } \\
\text { periodicamente }\end{array}$ & $\begin{array}{l}\text { Capacitação } \\
\text { ACS } \\
\text { as }\end{array}$ & $\begin{array}{c}\text { Enfermeiro } \\
\text { de EqSF }\end{array}$ & $\begin{array}{c}\text { ACS, mulheres } \\
\text { da comunidade } \\
\text { e escolares }\end{array}$ & $\begin{array}{c}\text { UBS } \\
\text { Pedro II/PI }\end{array}$ & $\begin{array}{c}\text { Oito } \\
\text { meses }\end{array}$ \\
\hline Saúde da mulher & Desenvolver & Realização & EqSF e & Mulheres de & UBS & SI \\
\hline
\end{tabular}




\begin{tabular}{|c|c|c|c|c|c|c|}
\hline $\begin{array}{l}\text { com ação } \\
\text { permanente de } \\
\text { prevenção contra o } \\
\text { câncer de colo de } \\
\text { útero e mama, } \\
\text { Gurupi/TO }\end{array}$ & $\begin{array}{l}\text { campanha para } \\
\text { realização do } \\
\text { exame } \\
\text { preventivo em } \\
\text { períodos } \\
\text { específicos e } \\
\text { inclusão do } \\
\text { tema em outras } \\
\text { campanhas } \\
\text { preventivas }\end{array}$ & $\begin{array}{l}\text { atividades } \\
\text { preventivas em que } \\
\text { se alerta a } \\
\text { necessidade dos } \\
\text { exames, por meio de } \\
\text { palestras, rodas de } \\
\text { conversas, teatros, } \\
\text { sorteios e salão de } \\
\text { beleza }\end{array}$ & $\begin{array}{l}\text { Coordenação } \\
\text { da Saúde da } \\
\text { Mulher da } \\
\text { SMS }\end{array}$ & 25 a 64 anos & Gurupi/TO & \\
\hline $\begin{array}{l}\text { "Eu me amo, eu me } \\
\text { cuido": prevenindo } \\
\text { o câncer de mama e } \\
\text { colo de útero - a } \\
\text { universidade em } \\
\text { parceria com a } \\
\text { comunidade }\end{array}$ & $\begin{array}{l}\text { Sensibilizar e } \\
\text { orientar } \\
\text { mulheres sobre } \\
\text { a prevenção do } \\
\text { CA de mama e } \\
\text { de colo de útero }\end{array}$ & 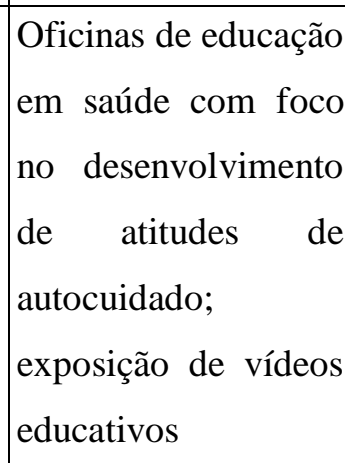 & $\begin{array}{l}\text { Acadêmicos } \\
\text { de medicina } \\
\text { da UFRN }\end{array}$ & Mulheres & $\begin{array}{c}\text { ESF } \\
\text { Pedra } \\
\text { Grande/RN }\end{array}$ & SI \\
\hline $\begin{array}{l}\text { Trazendo o rosa } \\
\text { para além do } \\
\text { outubro - cuidado } \\
\text { permanente em } \\
\text { saúde da mulher }\end{array}$ & $\begin{array}{l}\text { Alertar as } \\
\text { mulheres } \\
\text { quanto aos } \\
\text { sinais de alerta } \\
\text { do CA de colo } \\
\text { de útero e as } \\
\text { formas de } \\
\text { tratamento }\end{array}$ & 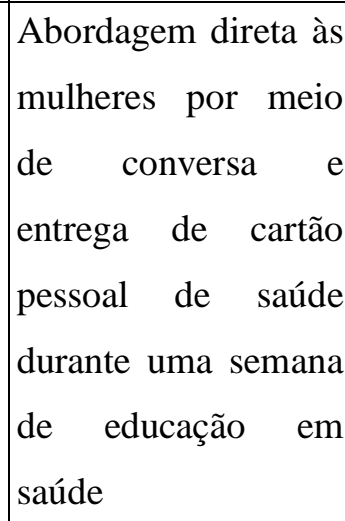 & SI & $\begin{array}{l}\text { Mulheres em } \\
\text { idade fértil }\end{array}$ & $\begin{array}{c}\text { UBS } \\
\text { Rocas/RN }\end{array}$ & $\begin{array}{c}\text { Uma } \\
\text { semana }\end{array}$ \\
\hline $\begin{array}{l}\text { Intervenção } \\
\text { multiprofissional } \\
\text { frente à saúde da } \\
\text { mulher }\end{array}$ & $\begin{array}{l}\text { Desenvolver } \\
\text { atividades de } \\
\text { prevenção na } \\
\text { APS }\end{array}$ & $\begin{array}{l}\text { Conversa com as } \\
\text { mulheres antes do } \\
\text { exame em espaço } \\
\text { protegido para que } \\
\text { possam tirar as suas } \\
\text { dúvidas e conhecer a } \\
\text { equipe que a atende }\end{array}$ & $\begin{array}{c}\text { Residentes em } \\
\text { saúde }\end{array}$ & Mulheres & \begin{tabular}{|c|} 
UBS \\
Dourados/MS
\end{tabular} & SI \\
\hline $\begin{array}{l}\text { Aprendendo a } \\
\text { aprender e a ensinar } \\
\text { na ESF: atividades }\end{array}$ & $\begin{array}{l}\text { Desenvolver } \\
\text { atividade } \\
\text { educativa sobre }\end{array}$ & $\begin{array}{l}\text { Palestra } \quad \text { com } \\
\text { apresentação } \quad \mathrm{em} \\
\text { slides, folhetos } \mathrm{e}\end{array}$ & $\begin{array}{c}\text { Acadêmicos } \\
\text { de medicina } \\
\text { da UFMS }\end{array}$ & $\begin{array}{c}\text { Frequentadores } \\
\text { do grupo de } \\
\text { planejamento }\end{array}$ & $\begin{array}{c}\text { UBS, Campo } \\
\text { Grande/MS }\end{array}$ & Um dia \\
\hline
\end{tabular}




\begin{tabular}{|c|c|c|c|c|c|c|}
\hline $\begin{array}{l}\text { educativas por } \\
\text { acadêmicos do } \\
\text { curso de medicina } \\
\text { da UFMS }\end{array}$ & anticoncepção & \begin{tabular}{|l} 
demonstração dos \\
métodos \\
anticoncepcionais
\end{tabular} & & familiar & & \\
\hline $\begin{array}{l}\text { Ação educativa de } \\
\text { atenção ao câncer } \\
\text { de colo uterino na } \\
\text { ESF Renascer em } \\
\text { Benevides/PA }\end{array}$ & $\begin{array}{l}\text { Sensibilizar as } \\
\text { mulheres acerca } \\
\text { da prevenção ao } \\
\text { CA de colo de } \\
\text { útero }\end{array}$ & $\begin{array}{llr}\text { Palestras, } & \text { brindes } & \text { e } \\
\text { vacinação } & \text { em } & \text { uma } \\
\text { ação } & \text { no } & \text { dia } \\
\text { internacional } & \text { da } \\
\text { mulher } & & \end{array}$ & $\begin{array}{l}\text { Enfermeiro } \\
\text { com EqSF }\end{array}$ & Mulheres & $\begin{array}{c}\text { Escola, } \\
\text { Benevides/PA }\end{array}$ & Um dia \\
\hline $\begin{array}{l}\text { A contribuição da } \\
\text { residência de } \\
\text { enfermagem } \\
\text { obstétrica na busca } \\
\text { ativa de portadoras } \\
\text { do vírus HPV em } \\
\text { uma UBS de } \\
\text { Campo Grande/MS }\end{array}$ & $\begin{array}{l}\text { Auxiliar na } \\
\text { busca ativa de } \\
\text { portadoras do } \\
\text { HPV }\end{array}$ & $\begin{array}{l}\text { Palestra sobre HPV } \\
\text { com os usuários que } \\
\text { se encontram na sala } \\
\text { de espera, incluindo } \\
\text { a distribuição de } \\
\text { materiais didáticos } \\
\text { impressos } \\
\text { preservativos }\end{array}$ & $\begin{array}{l}\text { Residentes em } \\
\text { enfermagem } \\
\text { obstétrica da } \\
\text { UFMS }\end{array}$ & $\begin{array}{l}\text { Usuários na } \\
\text { sala de espera }\end{array}$ & $\begin{array}{c}\text { UBS } \\
\text { Campo } \\
\text { Grande/MS }\end{array}$ & Um mês \\
\hline $\begin{array}{l}\text { Realize seu exame } \\
\text { citológico e } \\
\text { conquiste o sorriso } \\
\text { saudável }\end{array}$ & $\begin{array}{l}\text { Aumentar a } \\
\text { adesão ao } \\
\text { exame }\end{array}$ & $\begin{array}{l}\text { Campanha com o } \\
\text { tema "faça seu } \\
\text { exame preventivo e } \\
\text { fique com o sorriso } \\
\text { saudável", ofertando } \\
\text { uma profilaxia dental } \\
\text { para quem realizasse } \\
\text { o exame; palestras e } \\
\text { rodas de conversa } \\
\text { sobre a importância } \\
\text { do exame }\end{array}$ & SI & Mulheres & \begin{tabular}{|c|} 
UBS \\
Jaú de \\
Tocantins/TO
\end{tabular} & Um dia \\
\hline $\begin{array}{l}\text { Diálogo sobre o } \\
\text { HPV: perspectivas } \\
\text { de adesão ao } \\
\text { programa de } \\
\text { vacinação Anti- } \\
\text { HPV entre os pais } \\
\text { estudantes do PEJA }\end{array}$ & $\begin{array}{l}\text { Contribuir para } \\
\text { a divulgação e } \\
\text { adesão à } \\
\text { campanha de } \\
\text { vacinação } \\
\text { contra o HPV }\end{array}$ & $\begin{array}{l}\text { Rodas de conversa, } \\
\text { aula expositiva sobre } \\
\text { os sistemas genitais, } \\
\text { a confecção de } \\
\text { cartazes sobre o } \\
\text { HPV, vacinação e } \\
\text { pesquisas na internet }\end{array}$ & SI & $\begin{array}{l}\text { Alunos do } \\
\text { PEJA }\end{array}$ & $\begin{array}{c}\text { Escola do } \\
\text { PEJA } \\
\text { Rio de } \\
\text { Janeiro/RJ }\end{array}$ & SI \\
\hline
\end{tabular}




\begin{tabular}{|c|c|c|c|c|c|c|}
\hline & & $\begin{array}{ll}\text { pelos } & \text { participantes } \\
\text { sobre o } & \text { HPV e a sua } \\
\text { vacina } & \end{array}$ & & & & \\
\hline $\begin{array}{l}\text { Dia Rosa na UBS } \\
\text { Eloy de Souza em } \\
\text { Macaíba/RN }\end{array}$ & $\begin{array}{l}\text { Educar e } \\
\text { conscientizar } \\
\text { mulheres para a } \\
\text { mudança dos } \\
\text { padrões e } \\
\text { modos de vida }\end{array}$ & 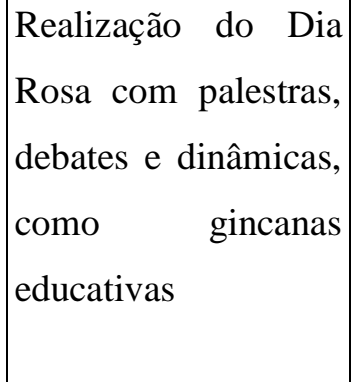 & $\begin{array}{l}\text { Acadêmicos } \\
\text { de medicina } \\
\text { da UFRN }\end{array}$ & Mulheres & $\begin{array}{c}\text { UBS } \\
\text { Macaíba/RN }\end{array}$ & Um dia \\
\hline $\begin{array}{l}\text { Introduzindo a } \\
\text { Promoção a Saúde } \\
\text { na UBS de Campo } \\
\text { da Mangueira em } \\
\text { Macaíba/RN }\end{array}$ & $\begin{array}{l}\text { Inserir práticas } \\
\text { de promoção a } \\
\text { saúde na rotina } \\
\text { de UBS }\end{array}$ & 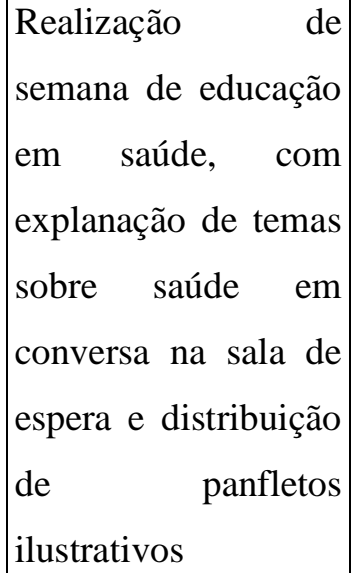 & $\begin{array}{l}\text { Acadêmicos } \\
\text { de medicina } \\
\text { da UFRN }\end{array}$ & $\begin{array}{l}\text { Usuários na } \\
\text { sala de espera }\end{array}$ & $\begin{array}{c}\text { UBS } \\
\text { Macaíba/RN }\end{array}$ & $\begin{array}{l}\text { Uma } \\
\text { semana }\end{array}$ \\
\hline $\begin{array}{l}\text { PET Saúde/Grupo } \\
\text { Medicina: } \\
\text { prevenção e } \\
\text { promoção em saúde } \\
\text { na UFSB }\end{array}$ & $\begin{array}{l}\text { Estimular a } \\
\text { comunidade } \\
\text { universitária a } \\
\text { pensar e cuidar } \\
\text { da sua saúde }\end{array}$ & $\mid \begin{array}{llr}\text { Criação } & \text { de } & \text { stands } \\
\text { com orientação sobre } \\
\text { prevenção } & \text { durante a } \\
\text { semana } & \text { do } & \text { dia } \\
\text { nacional } & \text { de } & \text { saúde, } \\
\text { com } & \text { oferta } & \text { de } \\
\text { práticas de cuidado e } \\
\text { entrega de folhetos }\end{array}$ & $\begin{array}{l}\text { Acadêmicos } \\
\text { de saúde da } \\
\text { UFSB e } \\
\text { profissionais } \\
\text { de saúde }\end{array}$ & $\begin{array}{l}\text { Comunidade } \\
\text { universitária }\end{array}$ & $\begin{array}{c}\text { UFSB } \\
\text { Teixeira de } \\
\text { Freitas/BA }\end{array}$ & Dois dias \\
\hline $\begin{array}{l}\text { Outubro rosa: } \\
\text { estratégia de } \\
\text { promoção e } \\
\text { fortalecimento para } \\
\text { a saúde da mulher }\end{array}$ & $\begin{array}{l}\text { Mobilizar } \\
\text { mulheres } \\
\text { quanto à } \\
\text { prevenção do } \\
\text { CA de colo de } \\
\text { útero }\end{array}$ & $\left|\begin{array}{lrr}\text { Campanha } & \text { incluindo } \\
\text { a } & \text { realização } & \text { de } \\
\text { fórum } & \text { sobre } & \text { saúde } \\
\text { da } & & \text { mulher, } \\
\text { divulgação } & \text { em } & \text { rádio } \\
\text { comunitária, } & \\
\text { caminhada } & \text { rosa, } \\
\text { disponibilização } & \\
\text { intensificada } & \text { do }\end{array}\right|$ & SI & Mulheres & $\begin{array}{l}\text { Nova Olinda } \\
\text { do Norte/AM }\end{array}$ & Um mês \\
\hline
\end{tabular}




\begin{tabular}{|l|l|l|l|l|l|l|}
\hline & $\begin{array}{l}\text { exame, atividades } \\
\text { educativas e } \\
\text { divulgação por carro } \\
\text { volante }\end{array}$ & & & \\
\hline $\begin{array}{l}\text { O NASF na } \\
\text { Atenção Básica de } \\
\text { Cedro de São } \\
\text { João/SE }\end{array}$ & $\begin{array}{l}\text { Desenvolver a } \\
\text { campanha do } \\
\text { Outubro Rosa } \\
\text { algumas atras e oferta de } \\
\text { de cuidado }\end{array}$ & Equipe NASF & Mulheres & $\begin{array}{c}\text { UBS e outros } \\
\text { Cedro de São } \\
\text { João/SE }\end{array}$ & Um mês \\
\hline
\end{tabular}

Fonte: Os autores (2017).

Legenda: ACS - Agente Comunitário de Saúde; APS - Atenção Primária à Saúde; CA - câncer; CRAS - Centro de Referência de Assistência Social; EqSF - equipe de saúde da família; ESF - Estratégia Saúde da Família; HPV - Papiloma Vírus Humano; NASF - Núcleo de Apoio à Saúde da Família; PEJA - Programa de Jovens e Adultos; SI - sem informação; SMS - Secretaria Municipal de Saúde; UBS - Unidade Básica de Saúde; UFMS Universidade Federal do Mato Grosso do Sul; UFRN - Universidade Federal do Rio Grande do Norte; UFSB Universidade Federal do Sul da Bahia.

Apesar de as mulheres adultas e idosas serem as diretamente atingidas pelo câncer de colo de útero e, inclusive, representar o público da maioria das ações aqui analisadas (Tabela 1), alguns fatores de risco podem ser trabalhados com todos os grupos populacionais, como a própria transmissão do Papilomavírus Humano (HPV), que se trata de uma Infecção Sexualmente Transmissível (IST), para o qual a adoção do sexo seguro trata-se de uma atividade preventiva importante. Nesse sentido, as ações relativas à sexualidade podem e devem ser realizadas também para os homens e nas escolas, de modo que possa ser diminuída a circulação do vírus na população, e, por conseguinte, diminuir a contaminação entre as mulheres sexualmente ativas.

Tabela 1 - Caracterização das ações

\begin{tabular}{l|l|c}
\hline \multicolumn{1}{c|}{ Dimensão } & \multicolumn{1}{c}{ Variável } & N \\
\hline Público-alvo das ações & Mulheres & 10 \\
& Usuários da UBS & 3 \\
& Comunidade escolar ou universitária & 3 \\
\hline & UBS & 10 \\
Localidades em que foram realizadas as ações & Escolas & Equipamentos diversos do território das UBS \\
& Campus universitário & 2 \\
\hline Responsáveis pela organização das ações & Profissionais de saúde & 1 \\
\hline
\end{tabular}




\begin{tabular}{lll}
\hline & Um ou dois dias & 5 \\
Duração das ações realizadas & Uma semana & 2 \\
& Um mês & 3 \\
& Oito meses & 1 \\
& Sem informação & 4 \\
\hline
\end{tabular}

Fonte: Os autores (2017).

Nota: uma das ações foi desenvolvida tanto com mulheres quanto escolares, por isso o total de 16.

Legenda: UBS - Unidade Básica de Saúde.

Dentre as ações analisadas, observa-se a tônica principal na realização do exame preventivo pelas usuárias do serviço, mesmo naqueles que enfatizavam outras questões (prevenção ao HPV, métodos anticoncepcionais, autocuidado etc.). O rastreamento trata-se de uma atividade de detecção precoce que deve ser incluída nas ações de prevenção ao câncer do colo do útero. $\mathrm{Na}$ realidade nacional, ele é realizado pelo teste Papanicolau (exame citopatológico do colo do útero), que deve ser ofertado às mulheres de 25 a 64 anos que já tiveram atividade sexual, sendo que os dois primeiros exames devem ser realizados com intervalo anual, e, se ambos os resultados forem negativos, os próximos devem ser realizados a cada três anos (INCA, 2016).

Kuschnir e Silva (2015) criticam que no Brasil o rastreamento ainda tem sido do tipo oportunístico, sendo realizado pelas mulheres que procuram o serviço de saúde. Isso tem gerado "um contingente de mulheres superrastreadas e outro contingente sem qualquer exame de rastreamento" (INCA, 2016, p. 32).

A Tabela 1 demonstra que as ações ainda são predominantemente desenvolvidas no interior dos serviços de saúde, com a própria população que os frequenta, como nas intervenções realizadas na sala de espera. Logo, a adesão ao rastreamento para detecção precoce desse câncer pelas mulheres que não os acessa periodicamente é um desafio. Nesse sentido, destaca-se a importância das ações que conseguem atingir um público para além dos já frequentadores dos serviços de saúde, como no relato que buscou a mobilização para a campanha com a criação de fóruns em um ambiente externo ao serviço de saúde, promoção de caminhada pelo território e divulgação da oferta do exame preventivo pela rádio comunitária e carro volante (DIAS, 2015). Em outro dos relatos, a atividade educativa, além de ser oferecida na própria unidade de saúde, foi realizada em um Centro de Referência de Assistência Social (CRAS) (SOARES, 2015). 
Em relação aos organizadores das ações, houve mais ações sendo desenvolvidas por estudantes, como graduandos e residentes de diversas áreas, seguido dos profissionais de saúde, como profissionais das EqSF e das equipes NASF e da gestão central da SMS.

O desenvolvimento de ações de promoção da saúde e prevenção de doenças pela APS ainda figura um desafio no contexto do SUS, havendo, por exemplo, pouca atuação no desenvolvimento de ações intersetoriais (ARANTES; SHIMIZU; MERCHÁN-HAMMAN, 2016). Isso pode ser identificado pelo baixo número de relatos encontrados na temática abordada em relação ao número total de registros, o que pode ter relação tanto pela própria gestão do trabalho que não favorece a realização desses tipos de ações, quanto pela própria formação dos profissionais de saúde, uma vez que apesar dos esforços de mudança consequentes em especial à expansão da ESF, o processo de formação dos profissionais persiste distante das necessidades do SUS de integração de conhecimentos clínicos e de saúde coletiva (BRASIL, 2007).

Segundo Romano (2008), a formação médica, o que também pode ser ampliado para as outras formações em saúde, ainda valoriza excessivamente a doença e o especialismo, sendo pequenos os investimentos nas dimensões subjetivas, preventivas e sociais para a prestação do cuidado. No entanto, o fato de haver muitas experiências desenvolvidas por universitários nos dados aqui analisados, seja na graduação ou na residência, demonstra um movimento das próprias universidades em traçar novas perspectivas formativas. A inclusão de experiências com ações de promoção e prevenção possibilita que esses estudantes tenham contato com esse tipo de ações, o que pode fazer com que tais atores se atentem sobre a sua importância na rotina do trabalho em saúde quando se inserirem futuramente nos serviços enquanto profissionais. Muitos desses relatos desenvolvidos pelo público universitário (e aqui analisados) foram decorrentes inclusive das próprias políticas de indução da reorientação de formação em saúde, como experiências no interior do Programa de Educação pelo Trabalho para a Saúde (PET-Saúde).

Todas as ações foram realizadas no âmbito dos municípios. 26,7\% das ações registradas na $\mathrm{CdP}$ foram desenvolvidas em municípios do Rio Grande do Norte, 20,0\% do Mato Grosso do Sul e 13,3\% do Tocantins. Já os estados do Amazonas, Bahia, Pará, Piauí, Rio de Janeiro e Sergipe tiveram cada um somente uma ação (6,7\%). Apesar do predomínio das ações do nordeste e norte brasileiro e não haver menção às iniciativas desenvolvidas em estados da região sul, isso não permite inferir que algumas regiões realizam mais atividades do que outras, uma vez que o registro das ações na CdP ocorre em caráter voluntário e por iniciativa dos próprios trabalhadores de saúde. 
Muitas ações foram pontuais, sendo que 60,0\% foram desenvolvidas em campanhas, algumas já consolidadas nacionalmente como o Outubro Rosa e Campanha de vacinação contra o HPV, e outras criadas nos locais, como o Dia Nacional de Saúde, Dia Rosa, Dia das Mães, Dia Internacional da Mulher, Mês da Mulher e Semana de Educação em Saúde (Tabela $1)$.

Esse predomínio de ações desenvolvidas por meio de campanhas pode ser encarado como uma herança presente nas práticas em saúde que são derivadas do modelo sanitarista da saúde pública institucionalizado no Brasil durante o século XX, que enfrentava os problemas de saúde da população mediante campanhas. Segundo Paim (1999), esse tipo de ação apresenta um caráter geralmente temporário e aparenta uma operação militar que objetiva o combate de uma epidemia, o controle de determinado agravo à saúde ou o esclarecimento e informação da comunidade sobre uma determinada questão.

A despeito da visibilidade e mobilização para um tema que as campanhas pretendem gerar, podendo propiciar maior ciência e conhecimento da população sobre uma determinada causa no momento em que são implementadas, vale trazer a crítica a tônica a qual elas são vinculadas, uma vez que elas são comumente usadas para estimular mudanças individuais de comportamento, embasadas pelo modelo biomédico. São influenciadas, portanto, "por uma ideologia política 'conservadora' que inclui pressupostos do tipo 'a insuficiência individual requer correção' e 'os riscos à ordem requerem controle', [...] [sendo] entendidas como 'mínimas' intervenções do Estado, ações que fornecem às pessoas informações 'para o seu próprio bem"” (OLIVEIRA, 2005, p. 427).

A persistência de atuação a partir desse modus operandi campanhista revela uma contradição entre as diretrizes e princípios do SUS e a concretização das práticas de cuidado nos cotidianos dos serviços de saúde. Apesar desse sistema ser institucionalizado a partir de um conceito amplo de saúde, há necessidade de maiores investimentos no desenvolvimento de ações que levem em conta o enfrentamento dos determinantes sociais da saúde (ARANTES; SHIMIZU; MERCHÁN-HAMMAN, 2016), ou seja, dos fatores sociais, econômicos, culturais e políticos que interferem nas condições de vida e saúde da população (BYDLOWSKI; WESTPHAL; PEREIRA, 2004).

Com vistas à análise das perspectivas pedagógicas presentes nas ações aqui analisadas, procedeu-se com a categorização a partir da classificação proposta por Bordenave (1989), que identifica três principais tendências: transmissão, condicionamento e problematização. Cada uma dessas perspectivas opera a partir de uma determinada ideologia e epistemologia, tendo implicações concretas sobre os sujeitos das ações. 
A perspectiva de transmissão predominou em oito dos quinze relatos analisados. Ela tem como premissa de que as ideias e os conhecimentos são os pontos mais importantes de um processo educativo. Os usuários são entendidos como "páginas em branco" nas quais os profissionais de saúde irão imprimir novas ideias e conhecimentos (BORDENAVE, 1989).

$\mathrm{Na}$ justificativa dos relatos analisados, os executores apostam na importância de se transmitir os conhecimentos acerca da doença e sua forma de prevenção por uma ausência de contato com essas informações:

A maioria das mulheres não tem conhecimento consolidado sobre as doenças, quanto a sua forma de transmissão/surgimento, prevenção e tratamento. A conversa pessoal com cada uma das usuárias tornou possível identificar as fragilidades individuais, desmistificar crendices e estigmas. (AMORIM; LOPES, 2015, não paginado).

[...] sempre buscando orientar sobre a importância da realização do exame anualmente, de como é realizada a coleta do material, além de tirar todas as dúvidas sobre gestação, métodos contraceptivos e Doenças sexualmente transmissíveis. (DST) (FLORES, 2015, não paginado).

[...] através dela [da campanha] estimulamos mulheres a conhecerem a realidade do câncer de mama, colo de útero, autocuidado e qualidade de vida. (DIAS, 2015, não paginado).

Desse modo, compreende-se a população como quem não sabe sobre o assunto e realizam-se ações que supram essa respectiva lacuna. Para atingir esse escopo, dentre as estratégias pedagógicas utilizadas nesses relatos, predominaram as palestras, conversas para esclarecimentos, utilização de banners e vídeos e entrega de materiais como panfletos e fôlderes:

Criamos panfletos ilustrativos sobre a saúde da mulher, hipertensão, hábitos de vida saudável e saúde da criança, nos quais abordamos temas como Câncer de Mama e colo do útero, alimentação saudável, vacinação, prática de exercícios físicos, hipertensão, diabetes, amamentação e saúde bucal. (MADRUGA, 2015, não paginado).

Czeresnia (2003, p. 55), a partir da crítica de que "a questão da educação não se resolve 'apenas' com informação e capacitação técnica", convoca para uma reconfiguração da educação/comunicação nas práticas de saúde. Isso se dá porque a própria compreensão de promoção da saúde "vai além de uma aplicação técnica e normativa, aceitando-se que não basta conhecer o funcionamento das doenças e encontrar mecanismos para seu controle" (CZERESNIA, 2003, p. 51-52). Esse tipo de ação poderia, pelo contrário, fortalecer a saúde por meio da construção da capacidade de escolha, conforme é possível observar na própria 
definição de promoção da saúde derivada da $1^{\circ}$ Conferência Internacional de Promoção da Saúde, realizada em Ottawa, Canadá, em 1986:

[...] processo de capacitação da comunidade para atuar na melhoria de sua qualidade de vida e saúde, incluindo uma maior participação no controle deste processo. Para atingir um estado de completo bem-estar físico, mental e social os indivíduos e grupos devem saber identificar aspirações, satisfazer necessidades e modificar favoravelmente o meio ambiente. A saúde deve ser vista como um recurso para a vida, e não como objetivo de viver. Nesse sentido, a saúde é um conceito positivo, que enfatiza os recursos sociais e pessoais, bem como as capacidades físicas. (BRASIL, 2002, p. 19-20).

Houve também ações que tiveram um caráter de condicionamento, em que a ênfase estava nos resultados comportamentais, ou seja, nas manifestações empíricas e operacionais relativas à troca de conhecimentos, atitudes e destrezas. Como uma justificativa para abordagens sob essa perspectiva, esteve a ideia de convencimento:

Gostaríamos de compartilhar esta experiência devido à dificuldade em convencermos as mulheres na faixa etária de 25 a 64 anos a realizar o exame papanicolau, visto que a cada dia nos deparamos com resultados de exames alterados. (CARDOSO, 2015, não paginado).

Desse modo, as atividades giraram em torno de estimular as mulheres para que realizassem o exame, como demonstrado nos objetivos presentes em alguns dos relatos:

[...] melhoria das condições de saúde através da educação e conscientização social, de modo a priorizar mudança dos padrões e modo de vida (CHAVES, 2015, não paginado).

Considerando a pequena conscientização da mulher sobre a prevenção ao câncer do colo de útero, desenvolveu-se esse projeto para proporcionar, conhecimento que desenvolva compromisso individual de realizar seus exames preventivos periodicamente e ser exemplo a outras mulheres da comunidade. (BARRETO, 2016, não paginado).

Conforme excerto acima, espera-se que o comportamento de algumas mulheres realizando o exame sirva de exemplo a outras, que, na visão da autora, serviria de estímulo para aumentar a realização do exame. Assim como a ideia de exemplo, outras estratégias foram utilizadas pelos relatos, que, para além das informativas que se assemelham à da perspectiva anteriormente analisada (como as palestras), incluíam sistemas de recompensa por meio de sorteios, entrega de brindes e gincanas, como a de perguntas e respostas de modo a avaliar se o conhecimento repassado havia sido fixado. Associou-se também a participação 
nas ações relativas ao câncer de colo de útero à possibilidade de realização de outros procedimentos na unidade:

Portanto em comemoração ao dia Internacional da Mulher realizamos uma campanha Citológica com o seguinte tema: Faça seu exame preventivo e fique com o sorriso saudável, pois após realização do exame a paciente ganhava uma profilaxia Dental, nesse dia realizamos palestras e rodas de conversas com temas sobre a importância do exame, e todas as mulheres que realizavam o exame ganhavam uma profilaxia dental assim incentivando as mesmas a adesão ao exame. (SOUZA, 2015, não paginado).

Desse modo, buscou-se criar um sistema de condicionamento, de modo que quem realizasse o exame preventivo - entendido aqui como a resposta esperada - pudesse ganhar alguma outra coisa em troca, que, no caso do relato anterior, era uma profilaxia dental recompensa pela resposta dada.

As experiências relacionadas a essas duas perspectivas revelam o baixo entendimento da população enquanto detentores de saberes, colocando em supremacia o conhecimento do profissional de saúde, que seria, considerado então, o detentor do saber verdadeiro:

A equipe de atenção básica, especialmente agente comunitário de saúde que irá conhecer a realidade do câncer de colo de útero no que diz respeito aos sintomas, fatores de risco, formas de tratamento disponíveis e estratégias de prevenção viabilizando à comunidade a disseminação da informação qualificada, pois sendo detentores do conhecimento, irão multiplicar e disseminar a informação através das visitas domiciliares e terão argumentos e conteúdos seguros para conquistar a comunidade a participar das ações na busca ao diagnóstico precoce do câncer de colo de útero. (BARRETO, 2015, não paginado).

Nesse sentido, o profissional argumenta no ato de "conquistar" a população, o que pode ser entendido como uma transformação dos sujeitos da sua ação como "quase 'coisa', num mero “isto"” (FREIRE, 2016, p. 186), o que revela o caráter antidialógico das duas perspectivas acima analisadas, apontando o verticalismo nessas práticas de saúde, que nega o conhecimento enquanto processo de busca.

Essas práticas de saúde sobre os comportamentos individuais tidos não saudáveis geram em seu processo uma culpabilização dos sujeitos pelo seu adoecimento (CARVALHO, 2004). O risco da penalização dos indivíduos, mediante o estabelecimento de critérios discutíveis de homogeneização de comportamentos, configura-se pela agudização do autoritarismo sanitário, o que diminui a margem de autonomia das pessoas, dos grupos humanos e das coletividades (BATISTELLA, 2007). 
Nesse sentido, atuar a partir da perspectiva da promoção da saúde deveria, pelo contrário, envolver o fortalecimento da capacidade individual e coletiva para lidar com a multiplicidade dos condicionantes da saúde, com enfoque na autonomia e na ação sobre os determinantes sociais. Desse modo, indo além de uma aplicação técnica e normativa, as ações de promoção da saúde não deveriam se restringir ao "conhecer o funcionamento das doenças e encontrar os mecanismos para seu controle" (CZERESNIA, 2003, p. 47-48), uma vez que abrange o "fortalecimento da saúde por meio da construção de capacidade de escolha, bem como à utilização do conhecimento como o discernimento de atentar para as diferenças e singularidades dos acontecimentos" (CZERESNIA, 2003, p. 48), ou seja, trata das singularidades e autonomia dos sujeitos.

Em contraponto as duas perspectivas acima apontadas, três dos relatos analisados optaram por realizar uma metodologia problematizadora, que, ao invés de se ater aos conhecimentos e ideias relevantes ou aos comportamentos corretos que se espera, busca aumentar a capacidade dos indivíduos para detectar os problemas reais e buscar para eles soluções originais e criativas (BORDERNAVE, 1989).

Dentre um dos expoentes dessa vertente pedagógica, está Paulo Freire, que, por meio de avanços teórico-conceituais e metodológicos, trouxe contribuições para se pensar a educação popular que gere uma pedagogia crítica e problematizadora. Algumas das experiências assumiram explicitamente o seu referencial: "Para o desenvolvimento do processo pedagógico escolheu-se a metodologia freiriana que possibilita o estudo de fatos reais e o envolvimento do aluno em um processo de reflexão e de conscientização (OLIVEIRA, 2015, não paginado)”.

Dentro dessa vertente, o diálogo é uma ferramenta fundamental de trabalho. Desse modo, isso exige, para além de espaços meramente informativos, instaurar encontros que busquem favorecer uma construção compartilhada de conhecimento a partir do encontro de saberes derivados entre a população e os profissionais de saúde, com diálogos entre os saberes científicos e os saberes populares.

Nesse sentido, tais ações foram permeadas de atividades que pudessem gerar maior horizontalidade do serviço de saúde e seus usuários, como oficinas, rodas de conversa e construção conjunta de materiais. No exemplo a seguir, houve a preocupação de desenvolver um processo que levasse os próprios participantes a refletirem sobre a sua realidade e atuassem de forma protagônica na ação: 
A estratégia de ensino contou com aula expositiva sobre os sistemas genitais; a confecção de cartazes sobre o HPV, a vacina Anti-HPV e o exame preventivo; pesquisas na internet sobre o HPV e a vacina Anti-HPV; e ainda de realizações de rodas de conversas. [...] Em resposta constatou-se os alunos do PEJA empenhados na solução de problemas extraídos de sua realidade social e integrados em um processo de reflexão crítica dos hábitos e atitudes, anteriormente assumidos. (OLIVEIRA, 2015, não paginado).

Esse processo faz com que todos os envolvidos cresçam e aprendam conjuntamente, afinal "ninguém sabe tudo, assim como ninguém ignora tudo" (FREIRE, 2015, p. 57), fazendo do processo educativo um processo coletivo de aprendizagem: "o educador problematizador refaz, constantemente, seu ato cognoscente, na cognoscibilidade dos educandos. Estes, em lugar de serem recipientes dóceis de depósitos, são agora investigadores críticos, em diálogo com o educador, investigador crítico, também” (FREIRE, 2016, p. 97).

Para que o diálogo seja instaurado nas práticas de saúde, faz-se necessário que os profissionais adquiram uma postura de escuta e de compreender a cultura popular também com uma forma de conhecimento, um conhecimento acumulado e sistematizado, que interpreta e explica a sua realidade (VALLA, 1990). Com essa postura diante das ações, é possível ser mais permeável a uma construção conjunta de conhecimento, que favorece também a instauração de um processo pedagógico mais dialógico, como avaliado nos relatos a seguir:

Esta oportunidade permitiu compreender a importância do planejamento para a sua realização bem como a aplicação, na prática, da dialogicidade, ou seja, de ouvir também os participantes. (FALCÃO, 2015, não paginado).

As oficinas foram uma oportunidade ímpar de compartilhamento de saberes sobre concepções de adoecimento, prevenção e cuidado. Aprendemos a ser profissionais que não apenas repassam informação, mas que escutam quem nos procura. (SANTANA, 2015, não paginado).

No entanto, compreensões como as acima referidas foram escassas nos relatos analisados. Isso faz com que as ações de promoção e de prevenção se afastem dos pressupostos da Política Nacional de Promoção da Saúde (BRASIL, 2014) e da Política Nacional de Educação Popular em Saúde (BRASIL, 2012). Oferecer atividades mais dialógicas que permitissem uma reflexão sobre o câncer de colo de útero e o seu enfrentamento poderiam favorecer maiores transformações individuais e sociais, estando orientadas a partir da perspectiva da educação popular em saúde, que pode ser entendida como uma: 
Prática político-pedagógica comprometida com o combate às desigualdades sociais e interessada em promover a participação dos indivíduos nas questões relativas à saúde e à vida da população. Constitui-se como um campo estratégico para a atuação dos que trabalham junto à população, cuja finalidade é inserir na sua prática cotidiana um espaço de reflexão da sua ação. A tarefa fundamental é a de agir junto com os usuários, como sujeito e não como objeto de transformação. (TRAVASSOS, 2016, p. 44).

O cenário aqui apresentado revela a importância de mais investimentos nos processos de educação permanente em saúde dos profissionais da atenção básica para que eles possam atuar a partir das diretrizes dessas políticas e de uma gestão do trabalho em saúde que seja mais sensível à participação popular nos diferentes aspectos relacionados ao processo saúdedoença-cuidado. A partir de estudo que avaliava a efetividade da atenção básica na detecção e prevenção do câncer em um município do interior de MG, Grillo (2013, p. 110) relatou inadequações semelhantes:

O acesso aos serviços básicos de saúde no âmbito do SUS, segundo nosso estudo apresenta-se limitado em alguns aspectos principais: a falta de capacitação dos profissionais das equipes da atenção básica (ESF); a baixa capacidade das equipes/ESF em elaborarem planos, programas, projetos e/ou ações contínuas para a prevenção e deteç̧ão precoce do câncer; a falta de conhecimento das políticas públicas e da doença "câncer".

Apesar de identificarmos a necessidade de formação permanente dos profissionais de saúde, foi possível perceber também que mesmo algumas atividades desenvolvidas por acadêmicos e residentes não atuavam em consonância com as diretrizes da promoção da saúde e da educação popular em saúde. Isso leva ao questionamento da própria importância dessas novas perspectivas sobre a produção do cuidado em saúde ser debatido e incluído na formação em saúde, que é marcada ainda por ser hegemonicamente biologicista, medicalizante e procedimento-centrada (CECCIM; FEUERWERKER, 2004).

\section{Considerações finais}

Os relatos de experiências aqui analisados demonstram ainda haver um predomínio de atividades pontuais, a partir de um modelo campanhista e com a tônica de se transmitir o conhecimento acerca da doença para população. Essas ações ocorreram predominantemente nas próprias unidades de saúde, o que ainda traz desafios para a prevenção do câncer de colo de útero, para o qual deveria haver maior mobilização para que ele deixasse de ser oportunístico. Para isso, é necessário engajar as mulheres que não frequentam 
sistematicamente as unidades de saúde a realizarem o exame preventivo na periodicidade recomendada.

A tímida inserção de atividades a partir de uma perspectiva problematizadora revela o quanto o ideário da promoção da saúde e da educação popular em saúde merece ser fortalecido nos serviços de saúde. Processos de educação permanente em saúde sobre essas temáticas, ambas institucionalizadas como políticas no interior do SUS, assim como sobre o modo como os profissionais de saúde desenvolvem suas ações preventivas e de promoção, poderiam gerar inovações no cotidiano da produção de saúde, com ações que resultassem em processos mais dialógicos, reflexivos e que possam atuar a partir de uma construção compartilhada de conhecimento. Nesse sentido, ainda figura um desafio para os profissionais de saúde articular o saber científico ao saber popular, bem como envolver mais a comunidade em suas ações, a partir de uma compreensão deles enquanto sujeitos - não identificá-los mais como meros depósitos de suas ações.

Apesar de ser um repositório com grande inserção nos serviços de saúde, houve algumas dificuldades para a análise a partir dos relatos de experiência da $\mathrm{CdP}$, dado que muitos desses registros eram deficitários de alguns detalhes sobre a ação relatada. Novas pesquisas que partam de outras fontes de dados, como a própria observação dessas práticas e entrevistas com esses profissionais, devem ser realizadas de modo a auxiliar na descrição e na análise das ações de promoção da saúde e prevenção do câncer de colo de útero, o que pode gerar melhorias em uma das etapas importantes do enfrentamento desse câncer no SUS, com grande impacto na qualidade de vida da população brasileira.

\section{Referências}

AMORIM, P.; LOPES, C. P. Trazendo o rosa para além do outubro: cuidado permanente em saúde da mulher. Comunidade de Práticas. Documento eletrônico. 2015. Disponível em: https://novo.atencaobasica.org.br/relato/4964. Acesso em: 10 set. 2017.

ARANTES, L. J.; SHIMIZU, H. E.; MERCHÁN-HAMMAN, E. Contribuições e desafios da Estratégia Saúde da Família na Atenção Primária à Saúde no Brasil: revisão de literatura.

Ciência \& Saúde Coletiva, Rio de Janeiro, v. 21, n. 5, p. 1.499-1.510, 2016. Doi: 10.1590/1413-81232015215.19602015.

BARRETO, K. A conscientização da mulher para fazer prevenção contínua ao câncer de colo de útero. Comunidade de Práticas. Documento eletrônico. 2016. Disponível em: https://novo.atencaobasica.org.br/relato/5386. Acesso em: 10 set. 2017. 
BATISTELLA, C. Abordagens contemporâneas do conceito de saúde. In: FONSECA, A. F.; CORBO, A. D. (org.). O território e o processo saúde-doença. Rio de Janeiro: EPSJV/Fiocruz, 2007. p. 51-86.

BORDENAVE, J. E. D. Alguns fatores pedagógicos. In: BRASIL. Ministério da Saúde. Secretaria de Modernização Administrativa e Recursos Humanos. Capacitação pedagógica para instrutor/supervisor área da saúde. Brasília: MS, 1989. p. 19-26.

BRASIL. Ministério da Saúde. Conselho Nacional de Secretários de Saúde. Atenção Primária e Promoção da Saúde. Brasília: CONASS, 2007.

BRASIL. Ministério da Saúde. Secretaria de Gestão Estratégica e Participativa. Comitê Nacional de Educação Popular em Saúde. Política Nacional de Educação Popular em Saúde. Brasília: MS, 2012.

BRASIL. Ministério da Saúde. Secretaria de Políticas de Saúde. Projeto Promoção da Saúde. As cartas da promoção da saúde. Brasília: MS, 2002.

BRASIL. Ministério da Saúde. Secretaria de Vigilância em Saúde. Secretaria de Atenção à Saúde. Política Nacional de Promoção da Saúde - PnaPS: Revisão da Portaria MS/GM n ${ }^{\circ}$ 687, de 30 de março de 2006. Brasília: MS, 2014.

BUSS, P. Promoção da saúde e qualidade de vida. Ciência e Saúde Coletiva, Rio de Janeiro, v. 5, n.1, p. 163-177, 2000. Doi: 10.1590/S1413-81232000000100014.

BYDLOWSKI, C. R.; WESTPHAL, M. F.; PEREIRA, I. M. T. B. Promoção da saúde. Porque sim e porque ainda não! Saúde e Sociedade, São Paulo, v. 13, n. 1, p. 14-24, 2004. Doi: 10.1590/S0104-12902004000100003.

CARDOSO, V. B. Saúde da mulher com ação permanente de prevenção contra o câncer de colo de útero e mama - Gurupi/TO. Comunidade de Práticas. Documento eletrônico. 2015. Disponível em: https://novo.atencaobasica.org.br/relato/11176. Acesso em: 10 set. 2017.

CARVALHO, S. R. As contradições da promoção à saúde em relação à produção de sujeitos e a mudança social. Ciência \& Saúde Coletiva, Rio de Janeiro, v. 9, n. 3, p. 669-678, 2004. Doi: 10.1590/S1413-81232004000300018.

CECCIM, R. B.; FEUERWERKER, L. C. M. Mudança na graduação das profissões de saúde sob o eixo da integralidade. Cadernos de Saúde Pública, Rio de Janeiro, v. 20, n. 5, p. 1.400-1.410, 2004. Doi: 10.1590/S0102-311X2004000500036.

CHAVES, B. Dia Rosa na Unidade Básica de Saúde Eloy de Souza em Macaíba, RN. Comunidade de Práticas. Documento eletrônico. 2015. Disponível em: https://novo.atencaobasica.org.br/relato/4967. Acesso em: 10 set. 2017.

CZERESNIA, D. O conceito de saúde e a diferença entre prevenção e promoção. In: CZERESNIA, D.; FREITAS, C. M. (org.). Promoção da saúde: conceitos, reflexões, tendências. Rio de Janeiro: Fiocruz, 2003. p. 39-53. 
DIAS, G. R. Outubro rosa: estratégia de promoção e fortalecimento para a saúde da mulher. Comunidade de Práticas. Documento eletrônico. 2015. Disponível em: https://novo.atencaobasica.org.br/relato/8429. Acesso em: 10 set. 2017.

FALCÃO, K. F. Aprendendo a aprender e a ensinar na Estratégia da Saúde da Família: atividades educativas por acadêmicos do curso de medicina da UFMS. Comunidade de Práticas. Documento eletrônico. 2015. Disponível em: https://novo.atencaobasica.org.br/relato/9527. Acesso em: 10 set. 2017.

FLORES, A. S. Intervenção multiprofissional frente à saúde da mulher. Comunidade de Práticas. Documento eletrônico. 2015. Disponível em: https://novo.atencaobasica.org.br/relato/10431. Acesso em: 10 set. 2017.

FREIRE, P. Extensão ou comunicação? Tradução de Rosiska Darcy de Oliveira. 17. ed. São Paulo: Paz e Terra, 2015.

FREIRE, P. Pedagogia do oprimido. 62. ed. Rio de Janeiro: Paz e Terra, 2016.

GRILLO, R. M. Política pública de saúde: a atenção básica na detecção precoce e prevenção do câncer. 2013. Dissertação (Mestrado em Serviço Social) - Faculdade de Ciências Humanas e Sociais, Universidade Estadual Paulista "Júlio de Mesquita Filho", Franca, 2013.

INCA - Instituto Nacional do Câncer. Coordenação de Prevenção e Vigilância. Divisão de Detecção Precoce e Apoio à Organização de Rede. Diretrizes brasileiras para o rastreamento do câncer do colo do útero. 2. ed. rev. atual. Rio de Janeiro: INCA, 2016.

INCA - Instituto Nacional do Câncer. Programa Nacional de Controle do Câncer do Colo do Útero. Rio de Janeiro: INCA, 2011.

KUSCHNIR, R. Organizando as Redes de Atenção à Saúde: perfis assistenciais, articulação entre níveis e organização de linhas de cuidado. In: KUSCHNIR, R.; FAUSTO, M. C. R. Gestão de Redes de Atenção à Saúde. Rio e Janeiro: EAD/ENSP, 2015. p. 129-168.

KUSCHNIR, R.; SILVA, L. B. Enfrentando o câncer de colo de útero. In: KUSCHNIR, R.; FAUSTO, M. C. R. Gestão de Redes de Atenção à Saúde. Livro 2. Rio de Janeiro: EAD/ENSP, 2015. p. 93-108.

LAVILLE, C.; DIONNE, J. A construção do saber: manual de metodologia da pesquisa em ciências humanas. Porto Alegre: Artmed; Belo Horizonte: Editora da UFMG, 1999.

MADRUGA, G. Introduzindo a promoção a saúde na UBS de Campo da Mangueira em Macaíba/RN. Comunidade de Práticas. 2015. Documento eletrônico. Disponível em: https://novo.atencaobasica.org.br/relato/4794. Acesso em: 10 set. 2017.

MINAYO, M. C. S. O desafio do conhecimento: pesquisa qualitativa em saúde. 14. ed. São Paulo: HUCITEC, 2014.

MORAES, M. M. S. et al. PET Saúde/Grupo Medicina: pevenção e promoção em saúde na Universidade Federal do Sul da Bahia-UFSB. Comunidade de Práticas. Documento eletrônico. 2017. Disponível em: https://novo.atencaobasica.org.br/relato/12597. Acesso em: 10 set. 2017. 
OLIVEIRA, D. L. A "nova” saúde pública e a promoção da saúde via educação: entre a tradição e a inovação. Revista Latino-Americana de Enfermagem, Ribeirão Preto, v. 13, n. 3, p. 423-431, 2005. Doi: 10.1590/S0104-11692005000300018.

OLIVEIRA, L. M. P. Diálogo sobre o Papilomavirus Humano: perspectivas de adesão ao programa de vacinação anti-HPV entre os pais estudantes do Programa de Ensino de Jovens e Adultos. Comunidade de Práticas. Documento eletrônico. 2015. Disponível em: https://novo.atencaobasica.org.br/relato/9799. Acesso em: 10 set. 2017.

PAIM, J. S. A reforma sanitária e os modelos assistenciais. In: ROUQUAYROL, M. Z.; ALMEIDA FILHO, N. (org.). Epidemiologia e Saúde. 5. ed. São Paulo: MEDSI, 1999. p. 474-487.

PARADA, R. et al. A política nacional de atenção oncológica e o papel da atenção básica na prevenção e controle do câncer. Revista de APS, Juiz de Fora, v. 11, n. 2, p. 199-206, 2008.

ROMANO, V. F. A busca de uma identidade para o médico de família. Physis, Rio de Janeiro, v. 18, n. 1, p. 13-25, 2008. Doi: 10.1590/S0103-73312008000100002.

SALES, N. A contribuição da residência de enfermagem obstétrica na busca ativa de portadoras do vírus HPV em uma Unidade Básica de Saúde de Campo Grande- MS. Comunidade de Práticas. Documento eletrônico. 2015. Disponível em: https://novo.atencaobasica.org.br/relato/10356. Acesso em: 10 set. 2017.

SANTANA, C. H. "Eu me amo, eu me cuido": prevenindo o câncer de mama e colo de útero - a universidade em parceria com a comunidade. Comunidade de Práticas. Documento eletrônico. 2015. Disponível em: https://novo.atencaobasica.org.br/relato/9455. Acesso em: 10 set. 2017.

SILVA, T. Ação educativa de atenção ao câncer de colo uterino na ESF Renascer em Benevides/PA. Comunidade de Práticas. Documento eletrônico. 2016. Disponível em: https://novo.atencaobasica.org.br/relato/11108. Acesso em: 10 set. 2017.

SOARES, R. O NASF na Atenção Básica de Cedro de São João/SE. Comunidade de Práticas. 2015. Documento eletrônico. Disponível em:

https://novo.atencaobasica.org.br/relato/5016. Acesso em: 10 set. 2017.

SOUZA, S. B. Realize seu exame citológico e conquiste o sorriso saudável. Comunidade de Práticas. Documento eletrônico. 2015. Disponível em:

https://novo.atencaobasica.org.br/relato/11244. Acesso em: 10 set. 2017.

TRAVASSOS, R. S. O trabalhador social em saúde. In: BORNSTEIN, V. J. et al. (org.). Curso de aperfeiçoamento em educação popular em saúde: textos de apoio. Rio de Janeiro: EPSJV, 2016. p. 43-44.

VALLA, V. V. A crise de interpretação é nossa: procurando compreender a fala das classes populares. Educação e Realidade, Porto Alegre, v. 21, p. 77-190, 1996.

WHO - Word Health Organization. Comprehensive cervical cancer control: a guide to essencial practice. 2. ed. Geneva: WHO, 2014. 
Submetido em $1^{\circ}$ de novembro de 2019.

Aprovado em 30 de abril de 2020. 